\title{
Multidimensional Sensorimotor Grounding of Concrete and Abstract Categories
}

\author{
Briony Banks $^{1 *}$ and Louise Connell ${ }^{1,2}$ \\ ${ }^{1}$ Department of Psychology, Lancaster University, Fylde College, Bailrigg, Lancaster, LA1 4YF, UK \\ ${ }^{2}$ Department of Psychology, Maynooth University, Maynooth, Co. Kildare, Ireland \\ ORCID: BB https://orcid.org/0000-0003-3666-0222; LC https://orcid.org/0000-0002-5291-5267
}

Keywords: abstract concepts, semantic categories, sensorimotor grounding, category production

\begin{abstract}
Summary
Semantic categories, and the concepts belonging to them, have traditionally been defined by their relative concreteness; that is, their reliance on perception. However, sensorimotor grounding must be regarded as going beyond the basic five senses, and incorporate a multidimensional variety of perceptual and action experience. We present a series of exploratory analyses examining the sensorimotor grounding of participantproduced member concepts for 117 categories, spanning traditionally concrete (e.g., animal, furniture) and highly abstract (e.g., unit of time, science) categories. We found that both concrete and abstract categories are strongly grounded in multidimensional sensorimotor experience. Both domains were dominated by vision and, to a lesser extent, head movements, but concrete categories were more grounded in touch and hand/arm action, while abstract categories were more grounded in hearing and interoception. Importantly, this pattern of grounding was not uniform, and subdomains of concrete (e.g., ingestibles, animates, natural categories, artefacts) and abstract (e.g., internal, social, non-social) categories were grounded in different sensorimotor dimensions. Overall, these findings suggest that the distinction between abstract and concrete categories is not as clearcut as ontological assumptions might suggest, and that the strength and diversity of sensorimotor grounding in abstract categories must not be underestimated.
\end{abstract}

\section{Introduction}

Categorisation is a fundamental part of human cognition, allowing us to group together distinct concepts and treat them as equivalent. However, most work on categorisation has traditionally concentrated on concrete categories (e.g., animals, fruit or tools), and our understanding of abstract categories (e.g., science, emotions or social relationships) is lacking in comparison. In particular, little is known about the specifics of how abstract categories are grounded in perceptual and action experience, and how their grounding compares to that of concrete categories.

Grounded (aka embodied) theories of semantic representation hold that concepts are grounded through our sensory and motor experiences in the world, via multiple sensory modalities (e.g., vision [1], touch [2], hearing [3], smell [4] and interoception [5]) and actions with different parts of the body (e.g., the face, arms and legs $[6,7])$. Accessing conceptual representations involves partial simulations of these perceptual and motor experiences: that is, some of the perceptual and motor systems involved in experiencing a concept are reactivated when, for example, reading the concept's label. Evidence for such theories now comes from a wide range of sources, including behavioural [2,8-11], neuroimaging [6,7,12,13], and neuropsychological studies $[3,14,15]$. Critically, sensorimotor simulations have been shown to be automatic and unconscious $[16,17]$ and intrinsic to our understanding of certain concepts $[18,19]$. Nevertheless, many theories assume that abstract concepts are more weakly grounded than concrete concepts [20-22].

Empirical work has shown, however, that abstract concepts are at least sometimes strongly grounded in perceptual and action experience. For example, the perceptual strength of a concept's dominant modality predicts lexical processing for abstract as well as concrete concepts [23, see also 24], while abstract words that evoke a richer sensory experience produce faster response times in semantic categorization [25]. Indeed, Banks et al. [26] found that sensorimotor information (composited across multiple dimensions) was equally predictive of responses in abstract category production (e.g., name as many members of the category science as possible) as in concrete category production (e.g., name as many animals as possible). Particular types of sensorimotor experience have also been associated with certain subdomains of abstract concepts. Interoceptive experience (i.e., sensations inside the body) is more important for abstract concepts than concrete, and particularly for emotion concepts [5]. Facial muscles are activated when making semantic decisions about emotion words [27], and the motor cortex is somatotopically recruited in emotion word processing in the same 
way as for action words [28]. Mouth movements are more important to abstract concepts than concrete [29,30], but are particularly associated with internal states and social concepts [31], while hand movements are particularly important to numerical and mathematical concepts [32-35]. Collectively, these findings suggest that abstract concepts may be almost as reliant on sensorimotor grounding as concrete concepts, but that the manner of this grounding (i.e., which perceptual modality or action effector) may depend on the exact type of abstract concept in question.

\section{The Current Study}

Despite the evidence summarised above, many questions remain regarding the grounding of semantic categories from both conceptual domains. Firstly, are categories of abstract and concrete concepts grounded to the same extent? Secondly, are all sensorimotor dimensions equally important for concrete and abstract categories, or does the type of grounding differ between the two domains? Traditionally, concrete concepts have been regarded as heavily reliant on vision and touch, but considering multiple dimensions of perception and action is important to fully understand their representation. Moreover, as noted above, the manner of grounding may differ between subdomains, particularly because the abstract domain is arguably more complex and heterogeneous than concrete [e.g., 33,36]. Thirdly, is sensorimotor grounding equally important to the cohesion of both abstract and concrete categories? Previous work has suggested that the members of abstract categories have less perceptual information in common than do the members of concrete categories [37], and hence do not rely on shared sensorimotor grounding in order to cohere as a category. Examining the grounding of concrete and abstract categories, across multiple sensory and motor dimensions, may provide insights into these questions.

In a series of exploratory studies, we therefore examined the multidimensional sensorimotor grounding of concrete and abstract categories generated from a large set of category production norms. Studying semantic categories via tasks such as category production (aka verbal fluency) provides a valuable source of information regarding the organisation and representation of conceptual knowledge and - because category members are freely produced by participants - does so with a higher degree of ecological validity than when researchers pre-select category members for analysis. While the majority of such research has focused on concrete categories [e.g., 38], recent category production norms by Banks \& Connell [39] cover a large number of categories in both abstract and concrete domains and thus form the basis of our present analyses. Operationalising multidimensional sensorimotor grounding, particularly for abstract concepts, is potentially complex. While some research on grounding has used concept feature lists [e.g., 20], we opted not to do so because it restricts data to aspects of representation that can be easily verbalised, not all of which have transparent sensorimotor grounding. Instead, we employed the Lancaster Sensorimotor Norms [40], which provide continuous ratings of experiential strength across 11 individual sensorimotor dimensions, where each dimension corresponds to a discrete area of sensory or motor cortical processing.

Our first aim was to compare the overall grounding of concrete and abstract categories (i.e., are they grounded in sensorimotor experience to the same extent? Study 1). Secondly, we compared the specific types of perceptual and action experience that are important across the two domains, and particularly for subdomains of both concrete and abstract categories (Study 2). Finally, we examine the diffuseness of concrete and abstract categories (i.e., within individual categories, how much do member concepts share sensorimotor information? Study 3). To this end, we analysed sets of member concepts spontaneously produced by participants for 117 concrete and abstract categories [39], using ratings of sensorimotor experience across 11 dimensions [40] to examine the multidimensional nature of concept grounding. As all analyses are exploratory, we report descriptive statistics only. All datasets and code for studies 1-3 are openly available [41].

\section{Study 1: Are abstract categories as strongly grounded as concrete categories?}

In our first study, we investigated whether concrete and abstract categories were grounded in sensorimotor experience to a similar extent, by examining how strongly their member concepts were experienced via perception and action.

\section{Method}

\section{Materials}

We analysed a set of category production norms [39] that comprised 67 concrete and 50 abstract categories. The 117 categories covered a range of taxonomic levels and category types (e.g. living vs. non-living, animate vs. inanimate, natural vs. artefact), including many categories frequently studied in the categorisation literature (e.g., animal, furniture, emotion) as well as more novel abstract categories (e.g., personal quality, statistical term), thus providing a large range of categories for comparison. We analysed a total of 2086 category member items (e.g., cat as a member of animal), excluding idiosyncratic items (i.e., category members produced by only one participant in the norms), for which sensorimotor ratings were available: 786 abstract category members and 1300 concrete category members (see [39] for full descriptive statistics).

To represent sensorimotor grounding, we used Lynott et al.'s Lancaster Sensorimotor Norms [40], where each concept was rated according to the extent to which it was experienced via six perceptual modalities (vision, 
hearing, taste, smell, touch, and interoception) and five action effectors (hand / arm, foot/leg, head, mouth, and torso). Specifically, we took a composite measure of sensorimotor strength for each item, Minowski-3 distance from the origin, which represented experience in all 11 dimensions but with an attenuated influence of weaker dimensions, and which Lynott et al. [40] found to be the best composite measure for predicting word recognition. Composite sensorimotor strength therefore ranged in theory from 0 (not experienced at all in any sensorimotor dimension) to 11.12 (experience greatly through all 11 sensorimotor dimensions). As Lynott et al.'s sensorimotor strength ratings were produced for American English, but our items from the Banks and Connell category production norms were for British English, we matched each item to its equivalent American English term in order to extract ratings. For example, we matched equivalent spellings (e.g., item theatre $\rightarrow$ ratings for theater) and dialectal terms (e.g., football $\rightarrow$ soccer; wrench $\rightarrow$ spanner). Plural items were also matched to singular equivalents (e.g., oranges $\rightarrow$ orange), abbreviated items were matched to their full version (e.g., sci-fi $\rightarrow$ science fiction), and quantifiers were ignored (e.g. one eighth $\rightarrow$ eighth). Polysemous items were matched to their category-specific sense if that term was available in the sensorimotor norms, (e.g., apple as a member of tree $\rightarrow$ apple tree; sprouts as a green vegetable $\rightarrow$ brussels sprouts). All matched terms are listed in the data file [41].

\section{Design and Analysis}

We explored the relative strength of sensorimotor grounding of abstract and concrete categories in two ways, first at a domain level by comparing sensorimotor strength of all items (i.e., all category members across all abstract vs. concrete categories), and then at a category level by calculating mean sensorimotor strength per category and ranking categories from weakest to strongest, observing where in the rank order abstract vs. concrete categories occurred.

\section{Results and Discussion}

Both abstract and concrete domains were strongly grounded in sensorimotor experience (see Figure 1), with very similar ranges of composite sensorimotor strength (1.71-8.06 for concrete, 1.55-7.45 for abstract). That is, both abstract and concrete categories had member concepts that were quite weakly (e.g., fortnight as a unit of time, hydrogen as a chemical element) and strongly (e.g., pain as a symptom of illness, shower as a bathroom fixture) grounded. On average, however, abstract categories had slightly lower sensorimotor strength $(M=$ $4.79, S D=1.0)$ than concrete categories $(M=5.23, S D=0.81)$; a difference of approximately $4.0 \%$ on the composite sensorimotor strength scale.

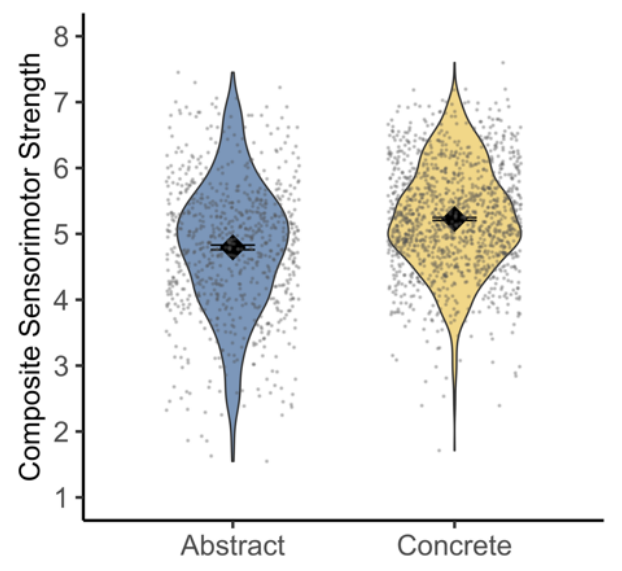

Figure 1. Composite sensorimotor strength of abstract and concrete category members. Black diamond shows the mean; error bars show \pm 1 SE.

When examining mean sensorimotor strength per category, the most strongly-grounded categories were concrete (e.g., green vegetable, fruit, mean rating > 6) and the weakest were abstract (e.g., day of the week, unit of time; mean rating $<3$ ). Nonetheless, the distinction between concrete and abstract categories was not as clear as might be expected (see Figure 2). Many abstract categories were ranked highly for their strong sensorimotor grounding (e.g., racket sport, symptom of illness, social gathering, art form, positive emotion), and were comparable in their mean sensorimotor strength to common concrete categories (e.g., vegetable, musical instrument, tool, furniture, farm animal). Similarly, many concrete categories were ranked low for their relatively modest sensorimotor strength (e.g., chemical element, snake, metal, gemstone, religious building) and were comparable to several clearly-abstract categories (e.g., religion, statistical term, military title, geometric shape).

These results indicate that both abstract and concrete categories are grounded in sensorimotor experience, albeit to a slightly weaker extent for abstract categories when considered as an overall domain, consistent with some previous findings [e.g., 23]. However, when the strength of sensorimotor grounding is considered for individual categories, the concrete-abstract dichotomy is no longer clearly apparent. 


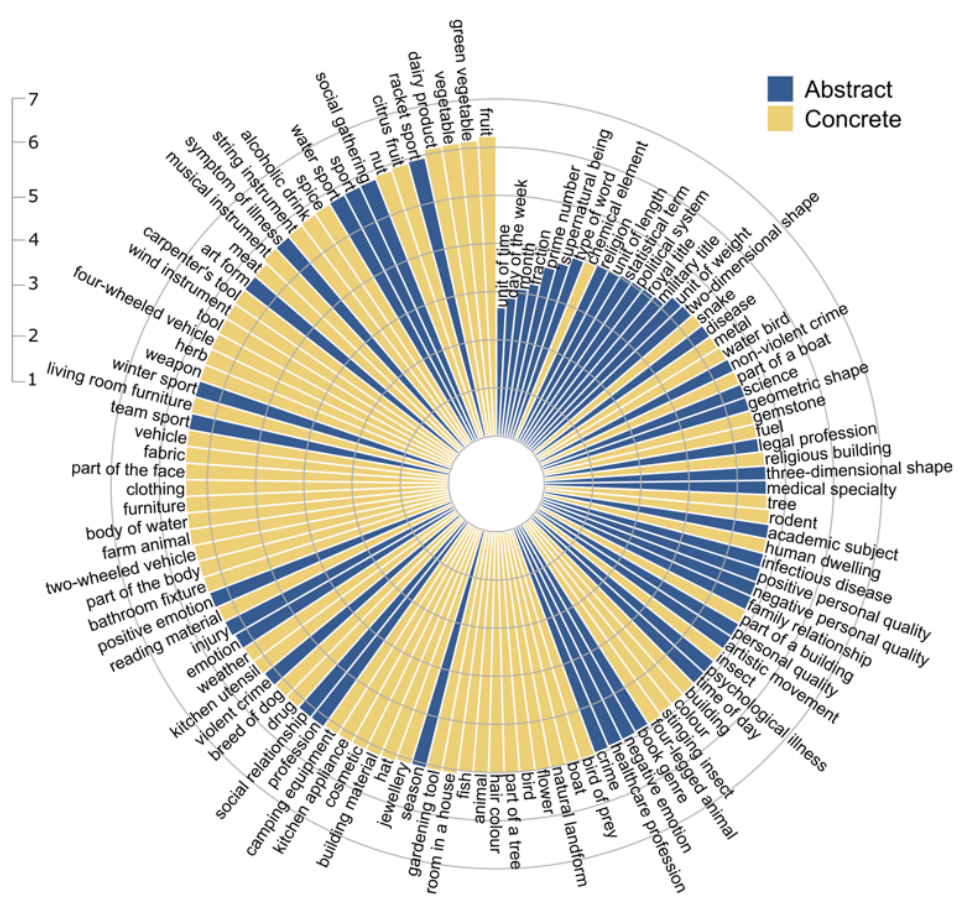

Figure 2. Mean composite sensorimotor strength per category, ordered clockwise from low to high.

\section{Study 2: Are abstract and concrete categories grounded via different sensory modalities and action effectors?}

Study 1 found that many abstract and concrete categories have equivalent strength of sensorimotor grounding. We next explored whether they differ in how they are grounded - that is, whether the overall domains are grounded via different perceptual modalities and / or different action effectors, and whether different subdomains of abstract and concrete categories have distinctive sensorimotor grounding.

\section{Method}

Materials

All items were the same as in Study 1. Rather than a single composite rating of sensorimotor strength to summarise grounding per item, we took Lynott et al.'s [40] ratings in 11 separate dimensions: perceptual experience via six modalities (vision, hearing, taste, smell, touch, and interoception) and action experience via five effectors (hand / arm, foot/ leg, head, mouth, and torso). Sensorimotor strength ratings ranged from 0 (not at all experienced in that dimension) to 5 (experienced greatly in that dimension).

\section{Design and Analysis}

We first explored broad differences at the domain level, comparing how abstract and concrete categories were grounded in each of the 11 sensorimotor dimensions. Our second exploratory goal was to examine subdomains of concrete and abstract categories, to establish whether different categorical types were differently grounded in perceptual and/or action experience. For concrete categories, we explored several candidate subdomains established in the neuropsychological literature (see [42] for a review) to represent the most common high-level dissociations in semantic deficits (i.e., living/non-living, animate/inanimate, biological/non-biological, natural/artefact, food/non-food), and separately examined others that are frequently ambiguous in their deficit patterns (i.e., musical instruments, parts of the body). We broadened the subdomain of food to cover anything that is taken into the body for ingestion (e.g., foodstuffs, drinks, drugs) and relabelled it as ingestible/non-ingestible for clarity. Note that while many of these subdomains largely overlap, they differ in the characterisation of certain categories (e.g., flowers are living, biological, and natural, but inanimate and non-ingestible; gemstones are natural but non-living, inanimate, non-biological, and noningestible). For abstract categories, we explored high-level subdomains that have been proposed in the literature to have different representational structure: internally-focused (i.e., relating to inner human experiences and characteristics) versus externally-focused categories (i.e., relating to experiences or entities outside the self), and social (i.e., relating to interpersonal interaction) versus non-social categories [31,33,43]. In both cases, we then allocated each semantic category from Banks and Connell [39] to its relevant subdomain (e.g., furniture in the inanimate subdomain; emotion in the internal subdomain), calculated the mean rating per dimension of all items within each subdomain, and examined differences in how each subdomain was grounded compared to its counterpart (e.g., animate vs. inanimate; internal vs. external). We selected the subdomains that showed the most distinctive differences in sensorimotor grounding, and then examined further nesting within each of these subdomains. 


\section{Results and Discussion}

Abstract and concrete category members share some similarities in how they are grounded (see Figure 3): both domains are primarily and strongly grounded in visual experience and (moderately) in head movements. Importantly, both domains are grounded to some extent across all 11 dimensions of perceptual and action experience, although to differing degrees. Abstract categories are more dependent than concrete categories on grounding in interoception, hearing, and movements of the mouth and head, as well as in - somewhat surprisingly - movements of the torso and foot/leg. By contrast, concrete categories are more dependent than abstract categories on grounding in haptic experience and hand/arm movements in particular, as well as in vision, smell and taste. Thus, although multiple dimensions of perceptual and action experience are important for both domains, abstract and concrete categories are overall grounded in different forms of sensorimotor experience.

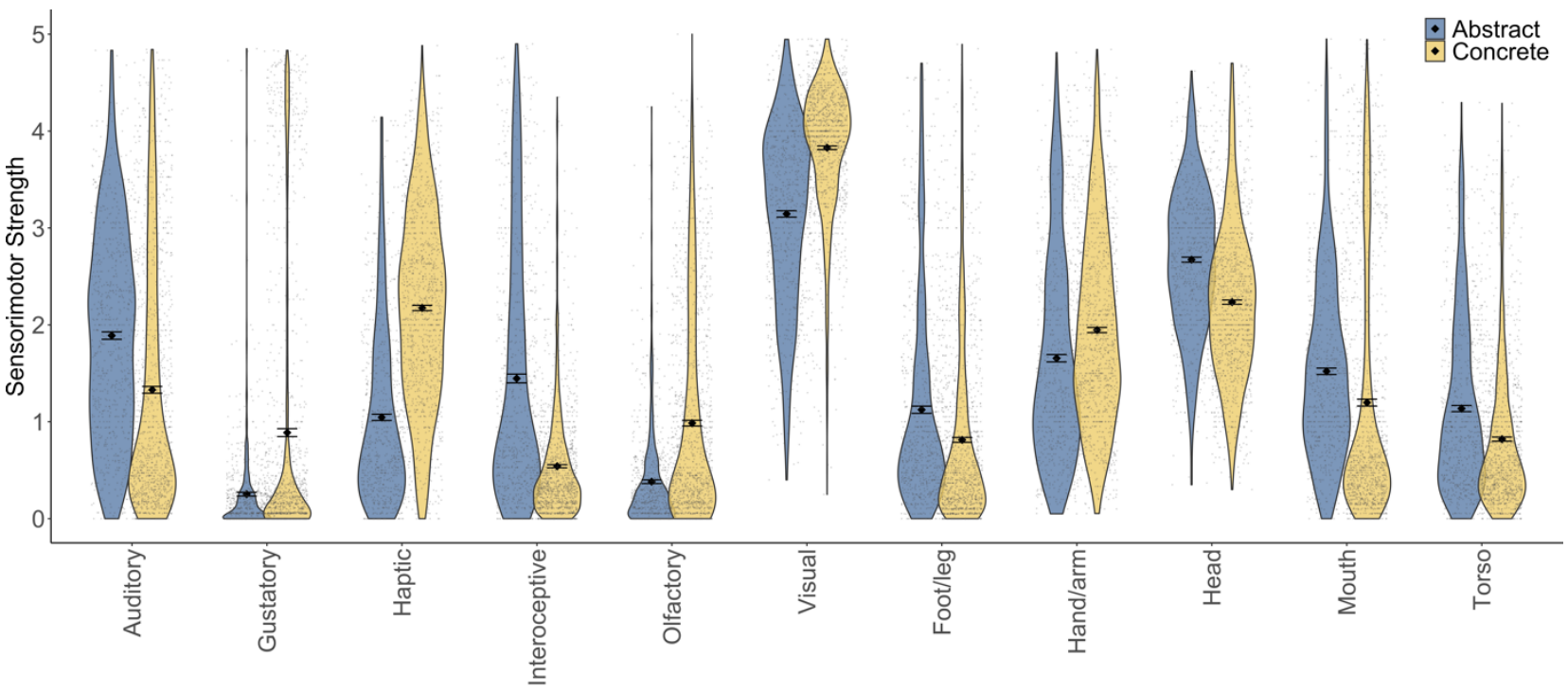

Figure 3. Sensorimotor strength ratings per dimension for abstract and concrete category members. Black diamonds show the mean rating; error bars show $\pm 1 S E$.

Figures 4 and 5 present different patterns of sensorimotor grounding for subdomains of abstract and concrete categories, respet. For abstract categories, all subdomains showed a consistently strong reliance on head action, but the clearest distinction was between internally- and externally-focused subdomains. Internal categories (e.g., emotion, personal quality, symptom of illness, injury, psychological illness) were most strongly grounded in interoception and head action, and it was the only abstract (or concrete) subdomain not dominated by visual experience. While some individual categories or member concepts in this subdomain relied on additional dimensions (e.g., injury is grounded in haptic and hand/arm action), the profile of internally-focused categories remained evident throughout. By contrast, external categories were most strongly grounded in vision and head action, with negligible grounding in interoception, and both social and non-social subdomains fit within this profile. Non-social categories (e.g., prime number, geometric shape, science, month, unit of weight) followed the same pattern of grounding that exemplifies external categories as a whole, with some individual categories also relying on additional dimensions (e.g., geometric shape was also grounded to a limited extent in haptic perception and hand/arm action). Social categories (e.g., family relationship, profession, religion, sport, crime) generally showed stronger visual and head-action grounding than the non-social subdomain, and were also strongly grounded in hearing and to some extent in mouth action. Again, some individual social categories were grounded in additional dimensions, such as sports reliance on hand/arm, foot/leg, and torso action, which was largely responsible for the presence of these latter dimensions in the abstract domain as a whole. 


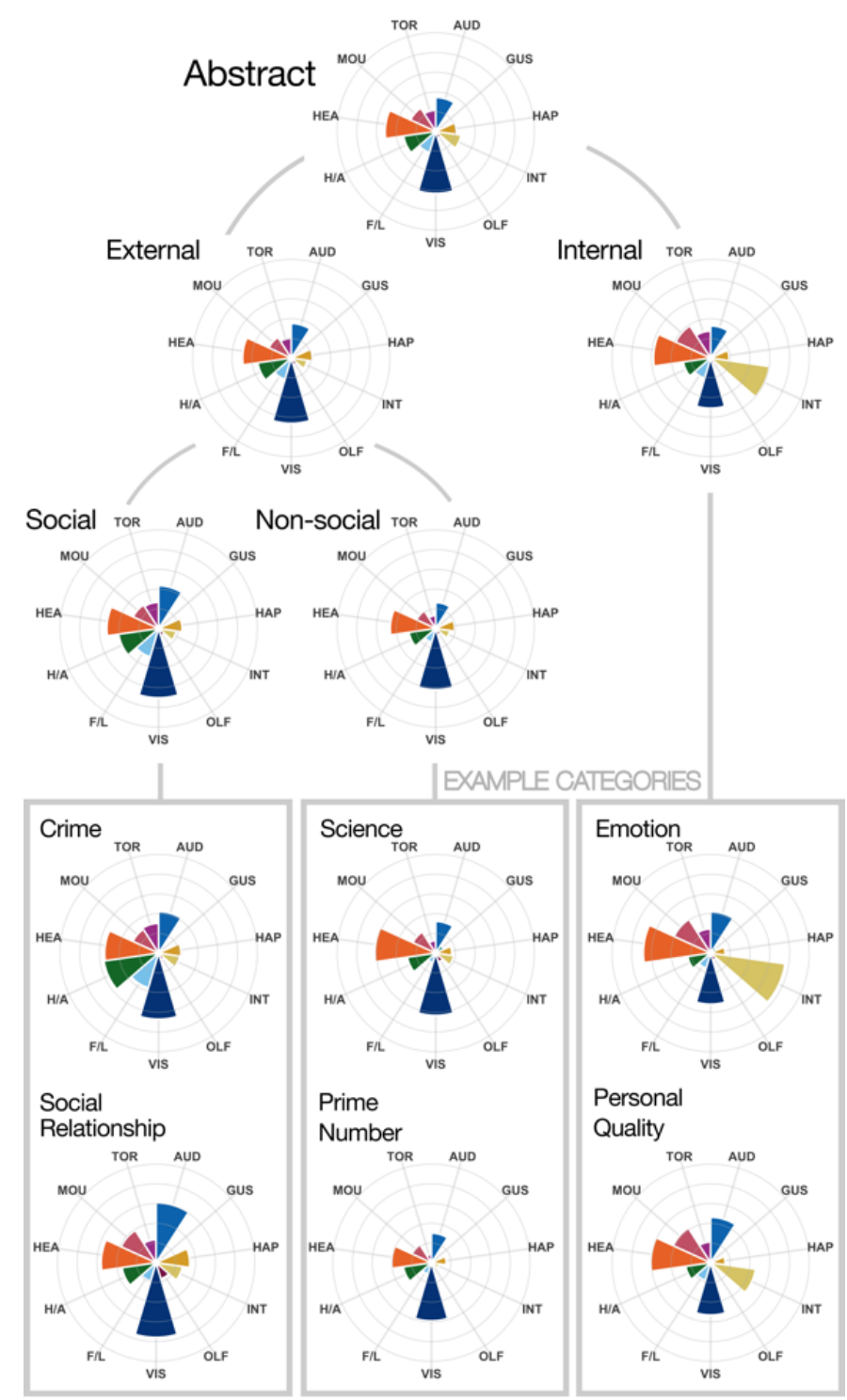

Figure 4. Mean ratings for 11 dimensions of sensorimotor experience for abstract categories. AUD = auditory; GUS = gustatory; HAP = haptic; INT = interoceptive; OLF = olfactory; VIS = visual; F/L = foot/leg; H/ A = hand $/$ arm; HEA = head; MOU = mouth; TOR = torso.

For concrete categories, all subdomains were dominated by grounding in vision and a consistent, moderate presence of head action. The most striking differences in grounding appeared between things that can be ingested (i.e., all food categories such as fruit, vegetable, meat, dairy product, plus alcoholic drink and drug) and things that cannot, which produced larger distinctions than any other candidate subdomains (see supplementary materials). Ingestible categories relied strongly on gustatory, olfactory, and mouth/throat action experience (in addition to the general concrete reliance on vision and head action), and to some extent on haptic and hand/arm action, but tended to have negligible grounding in auditory or foot/leg action. Two ingestible categories (alcoholic drink, drug) were additionally grounded in interoception, but otherwise followed the pattern of the subdomain. On the other hand, non-ingestible categories resembled the overall profile of concrete categories in being dominated by vision and head action, with a moderate grounding in haptic and hand/arm action that was stronger than that exhibited by ingestible categories, and a notable absence of grounding in taste or interoception. Examining non-ingestible categories more closely showed a clear distinction between animate and inanimate subdomains. Animate categories (e.g., animal, bird, snake, insect) were dominated by auditory grounding in addition to the ubiquitous visual and head action grounding, and had a markedly weaker reliance on touch and hand/arm action. By contrast, inanimate categories followed the pattern of the non-ingestible domain, and further subdivided into natural/artefact subdomains. Natural categories (e.g., flower, tree, body of water, weather) were overall dominated simply by visual and head-action grounding, with a weaker reliance on touch and hand/arm action than the inanimate subdomain as a whole. Some individual natural categories were also grounded in additional sensorimotor dimensions (e.g., flower and body of water had some olfactory grounding; natural landform had some foot/leg grounding) but never as the dominant means of grounding. Artefact categories (e.g., clothing, furniture, tool, vehicle), however, generally followed the overall profile of the inanimate subdomain but with a greater reliance on touch and hand/arm action in addition to vision and head action. Again, while some 
individual categories or member concepts within the artefact subdomain featured additional grounding in other sensorimotor dimensions (e.g., vehicle and weapon had some auditory grounding; vehicle and clothing had some foot/leg grounding), the characteristic profile of the subdomain was generally present throughout.

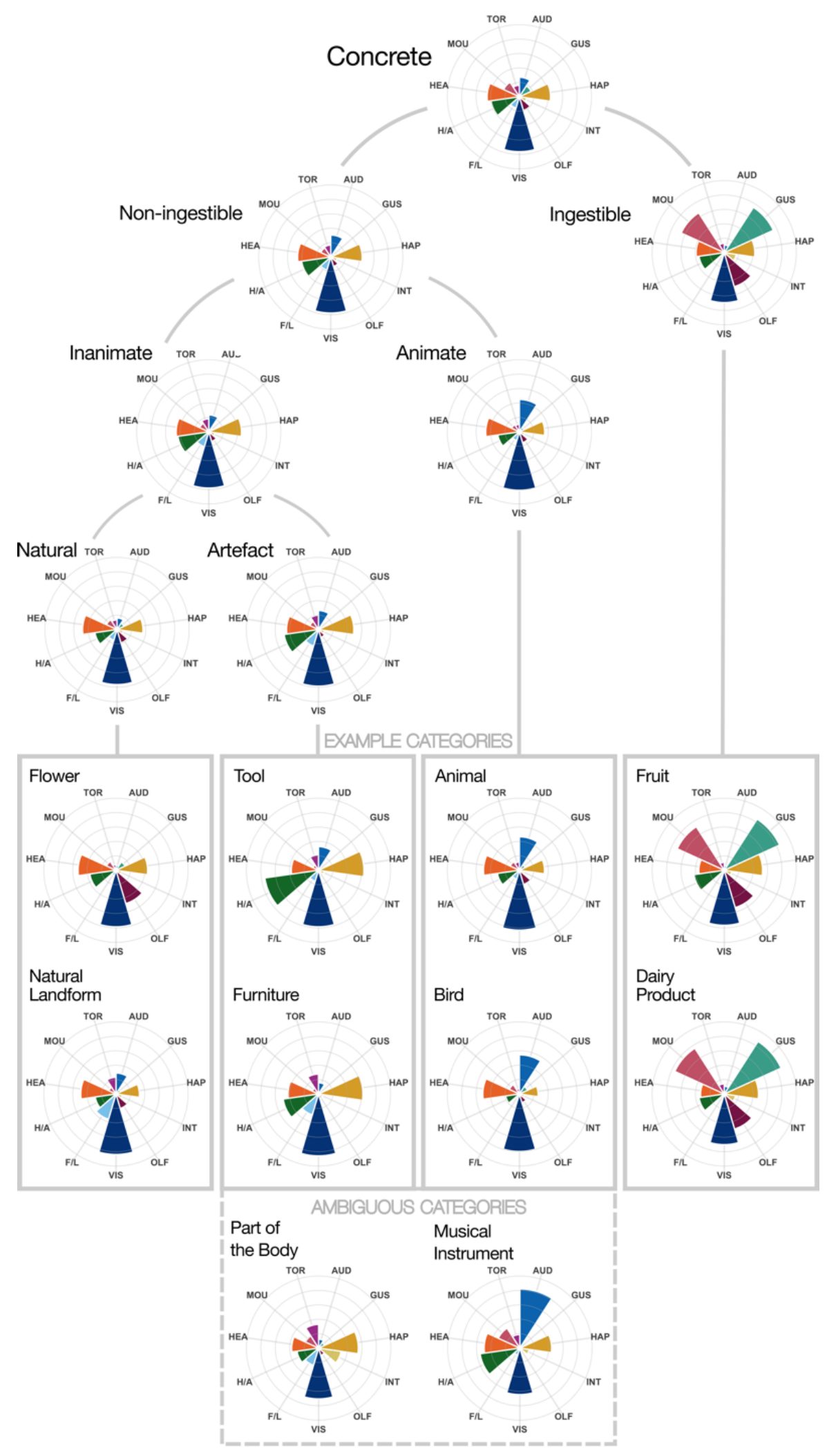

Figure 5. Mean ratings for 11 dimensions of sensorimotor experience for concrete categories. AUD = auditory; GUS = gustatory; HAP = haptic; INT = interoceptive; OLF = olfactory; VIS = visual; F/L = foot / leg; H/ A = hand / arm; HEA = head; MOU = mouth; TOR = torso. 
Lastly, we turned our attention to the ambiguous concrete categories of parts of the body and parts of the face, which are often assumed to be animate categories but can be impaired or preserved alongside inanimate artefacts in neuropsychological studies [e.g., 44]. In our data, the sensorimotor profiles for parts of the body/face more closely resembled the artefact subdomain in their strong haptic but weak auditory grounding. The categories of musical instruments (i.e., musical instrument, wind instrument, string instrument) represent another ambiguous case, in that they are technically artefacts and are sometimes impaired or preserved as such [e.g., 45], but often instead pattern after animate categories [e.g., 46]. We found that the sensorimotor profiles of musical instruments resembled a hybrid of both subdomains, being similar to the animate subdomain in their strong auditory grounding, but also similar to the artefact subdomain in their strong haptic and hand-arm grounding. Overall, the distinct patterns we observe in these analyses support previous findings that abstract and concrete categorical domains are both strongly grounded in sensorimotor experience [5,23], but - critically - not with a uniform pattern across different subdomains. Moreover, different subdomains of abstract and concrete categories can be characterised by their distinctive profiles of sensorimotor grounding.

\section{Study 3: Are abstract categories more diffuse than concrete categories?}

Studies 1-2 showed that abstract and concrete categories differ little in terms of the strength of their grounding, although they are grounded in very different types of sensorimotor experience. However, one other important difference may exist between the domains regarding the extent to which sensorimotor grounding contributes to the ability of member concepts to cohere as a category, as previous work has proposed that the members of concrete categories appear to share more overlapping perceptual information than do the members of abstract categories[37]. That is, abstract categories may be more diffuse in their sensorimotor grounding than concrete categories, whereby their member concepts may share relatively little sensorimotor experience in common, and hence are unlikely to rely on grounding for category cohesion. In this final study, we explored the overlap of sensorimotor experience between category members within each individual category in the abstract and concrete domains.

\section{Method}

Materials

All materials were the same as in Study 2, where each category member is represented as an 11-dimensional vector of sensorimotor experience.

\section{Design and Analysis}

To examine the diffuseness of categories, we used a measure of sensorimotor distance between all the members that comprised each category. For example, for the category animal, we calculated the distance between cat and all other members of that category (e.g., dog, lion, rhino, ...) and then did the same for all other category members (e.g., dog compared to lion, rhino, ...). Specifically, we calculated the sensorimotor distance between category members as the Minkowski-3 distance between their vectors [47], and then calculated the mean inter-member distance for that category. This final measure per category thus reflects the overlap of sensorimotor experience between its member concepts, and indicates how 'diffuse' the internal structure of each category is in its sensorimotor grounding. Mean sensorimotor distance per category ranged in theory from 0 (i.e., all category members are identical; category is not at all diffuse) to 11.12 (i.e., all category members share no sensorimotor information; category is extremely diffuse).

Our exploratory goal in this study was to examine whether abstract and concrete categories differed in their level of diffuseness by comparing the distribution of mean sensorimotor distance per category in each domain.

\section{Results and Discussion}

Most categories were not very diffuse in their grounding and mean sensorimotor distance between category members differed very little between the two domains (see Figure 6). Indeed, concrete categories $(M=2.26, S D$ $=0.56)$ were very slightly more diffuse than abstract categories $(M=2.23, S D=0.61)$; that is, within concrete categories, member concepts overlapped slightly less in sensorimotor experience than did the member concepts of abstract categories. In terms of sensorimotor grounding, the most diffuse categories were found in both domains (e.g., art form, weather). One particular concrete category, part of the body, was an outlier in its diffuse grounding, whereby its member concepts tended to be dominated by different sensorimotor dimensions (see Figure 7). Many of the least diffuse categories were also found in both domains (e.g., religion, gemstone), although the absolute lowest-scoring categories were predominantly abstract (e.g., 2-dimensional shape; type of word). The members of such non-diffuse categories tended to be grounded in the same sensorimotor dimensions and to similar extents, as illustrated in Figure 7 for members of the category religion. 


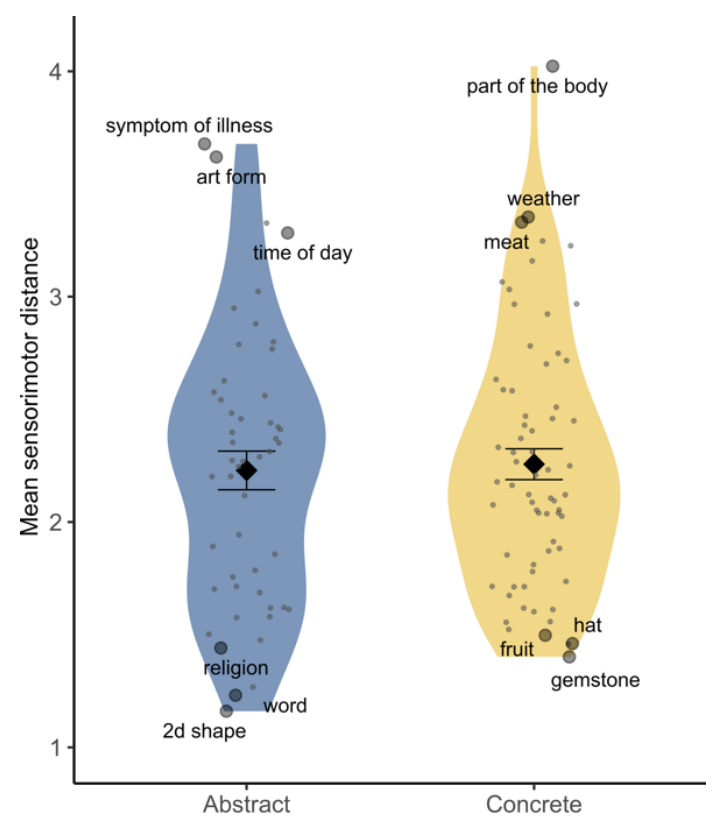

Figure 6. Mean sensorimotor distance between category members for each category, with examples of the most and least diffuse categories labelled by name. Black diamond shows the mean across all categories; error bars show $\pm 1 S E$.
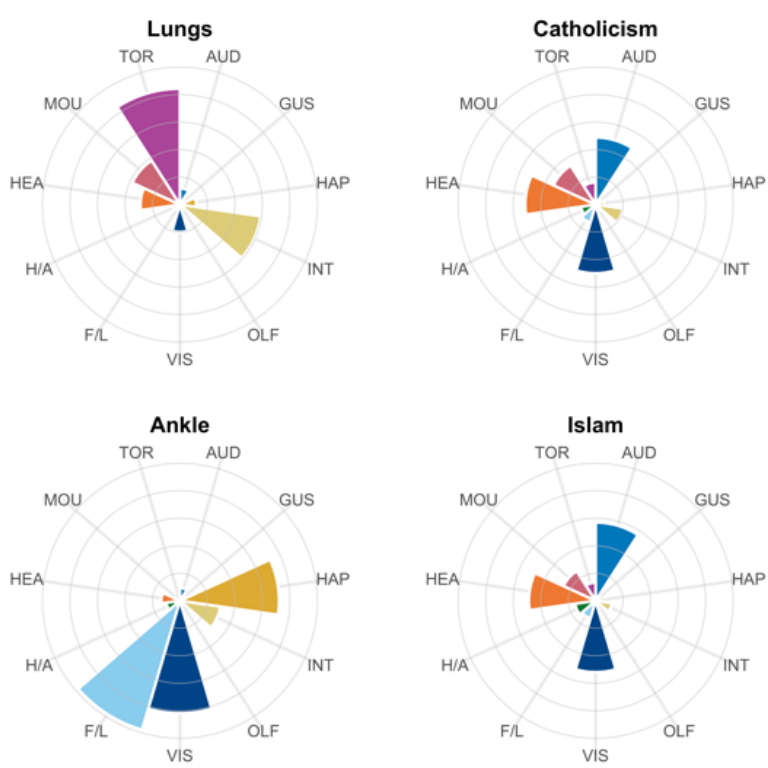

Figure 7. Multidimensional profile of sensorimotor strength ratings for sample member concepts from one of the most diffuse categories (part of the body, left) and least diffuse categories (religion, right).

Since a handful of categories were relatively diffuse in their sensorimotor grounding, it begs the question: what makes these categories cohere if their member concepts share little sensorimotor experience in common? Kloos and Sloutsky [37] proposed that such categories cohere on the basis of inclusion rules that are true for members and false for non-members. A similar proposal is that such categories are relational [e.g., 48] - that is, their members are grouped together because they share relational roles or patterns in common rather than because they share intrinsic properties such as sensorimotor experience. Some of the more diffuse categories in our data could indeed be considered to follow a rule or shared relation. For instance, every member of the category art form occupies the result role in an act of creation by an artist (similarly, every member of part of the body occupies a part-of relation with the body). However, many non-diffuse categories could also be considered rule-based or relational (e.g., prime number, human dwelling), so it is not the case that rules or relations replace sensorimotor grounding as a means of category coherence. Nonetheless, it is possible that rules or relational connections, perhaps expressed by language [e.g., 49], help categories with diffuse sensorimotor grounding to cohere more firmly.

Overall, abstract and concrete categories are about as diffuse as each other in their sensorimotor grounding; that is, categories in each domain are composed of member concepts that generally overlap to the same extent in their reliance on various perceptual modalities and/or action effectors. As a result, sensorimotor grounding 
is approximately equally important to category coherence in both domains. This pattern is not consistent with Kloos and Sloutsky's [37] suggestion that abstract categories would be more diffuse (i.e., their members overlapping less in perceptual information) than concrete categories. However, it should be noted that their proposal was primarily based on counting discrete features in artificial categories and hence may not generalise well to the continuous measures of sensorimotor grounding in real-world semantic categories that we examine here.

\section{General Discussion}

In three studies exploring the multidimensional sensorimotor grounding of abstract and concrete categories, we found many similarities between the two domains. Categories from both domains were strongly grounded in multiple dimensions of perceptual and action experience, with many categories and their member concepts having equivalent strength of sensorimotor experience. Both concrete and abstract categories were most strongly grounded in vision and had a moderate but consistent grounding in head action. Indeed, abstract and concrete categories were equally diffuse in their grounding, whereby category members overlapped with each other's sensorimotor experience to a similar extent, meaning that sensorimotor grounding was equally important to category coherence in both domains. Overall, we found that abstract and concrete categorical domains were more similar than might be predicted by theories stating that sensorimotor experience is more important to concrete concepts [20-22].

The most notable differences we observed were in the importance of different sensorimotor dimensions to each domain. Haptic experience and hand/arm movements, and to some extent smell and taste, were far more important to concrete categories than abstract, while interoception, hearing and to some extent head movements, were more important for abstract categories. This pattern supports and extends findings from the existing literature regarding the sensorimotor basis of abstract concepts [5,31], but also highlights that concreteness does not necessarily reflect the presence or absence of sensorimotor grounding; rather, it indicates the type of grounding typically associated with a domain.

Importantly, our analyses also identified grounding differences in subdomains of both abstract and concrete categories. Supporting theoretical distinctions between subdomains already suggested by the literature [e.g., $31,33,43]$, abstract categories fall into subdomains of internally-focused (typified by interoceptive and headaction grounding) and externally-focused (typified by visual and head-action grounding, without interoception), the latter of which can further split into social (typified by additional auditory grounding) and non-social subdomains. Similarly, concrete categories fall into subdomains of ingestible (typified by strong grounding in taste, smell, vision, and mouth action, and to some extent in touch and head action) and noningestible (typified by visual, haptic, head-action and hand-action grounding, and an absence of taste). Noningestible in turn falls into further subdomains of animate (typified by additional auditory grounding and weaker haptic and hand/arm grounding) and inanimate, the latter of which subdivides into natural (typified by visual and head-action grounding, with relatively weak haptic and hand/arm grounding) and artefact subdomains (typified by touch and hand/arm action in addition to visual and head-action grounding). Body parts more closely resembled artefacts, and musical instruments resembled both animate and artefact subdomains. Our findings for concrete categories thus mirror some of the patterns shown by patients with selective categorical deficits in semantic memory [e.g., 42,44] purely on the basis of sensorimotor strength (i.e., without functional or other features).

The present findings overall indicate that the concrete-abstract dichotomy is not the optimal way to understand grounding of semantic categories or conceptual representation in general, an argument which has been put forward by several researchers in recent years [5,31,43]. Indeed, defining concepts only in terms of their reliance on visual and haptic experience (e.g., as predominantly captured in single-dimension ratings such as imageability [23]) may bias our understanding of the conceptual system and its structure by providing a false impression of a fundamental sensory dichotomy between the domains. As we have demonstrated, examining more fine-grained differences between concepts and categories, based on multiple sensorimotor dimensions, can be particularly revealing for how categorical domains are represented. Far from being detached from the world of perception and action, many abstract categories relate just as richly to our sensory and motor experiences as do concrete categories.

\section{Acknowledgments}

This research was funded by the European Research Council (ERC) under the European Union's Horizon 2020 research and innovation programme (grant agreement no. 682848) to LC.

\section{References}

1. Zwaan RA, Stanfield RA, Yaxley RH. 2002 Language Comprehenders Mentally Represent the Shapes of Objects. Psychol. Sci. 13, 168-171. (doi:10.1111/1467-9280.00430)

2. Connell L, Lynott D, Dreyer F. 2012 A Functional Role for Modality-Specific Perceptual Systems in Conceptual Representations. PLoS ONE7, e33321. (doi:10.1371/journal.pone.0033321)

3. Bonner MF, Grossman M. 2012 Gray matter density of auditory association cortex relates to knowledge of sound concepts in primary progressive aphasia. J. Neurosci. Off. J. Soc. Neurosci. 32, 7986-91. (doi:10.1523/JNEUROSCI.6241-11.2012) 
4. González J, Barros-Loscertales A, Pulvermüller F, Meseguer V, Sanjuán A, Belloch V, Ávila C. 2006 Reading cinnamon activates olfactory brain regions. Neurolmage32, 906-912. (doi:10.1016/j.neuroimage.2006.03.037)

5. Connell L, Lynott D, Banks B. 2018 Interoception: the forgotten modality in perceptual grounding of abstract and concrete concepts. Philos. Trans. R. Soc. B Biol. Sci. 373. (doi:10.1098/rstb.2017.0143)

6. Hauk 0, Johnsrude I, Pulvermüller F. 2004 Somatotopic Representation of Action Words in Human Motor and Premotor Cortex. Neuron 41, 301-307. (doi:10.1016/50896-6273(03)00838-9)

7. Pulvermüller F, Hauk 0, Nikulin VV, IImoniemi RJ. 2005 Functional links between motor and language systems. Eur. J. Neurosci. 21, 793-797. (doi:10.1111/j.1460-9568.2005.03900.x)

8. Connell L, Lynott D. 2010 Look but don't touch: Tactile disadvantage in processing modality-specific words. Cognition 115, 1-9. (doi:10.1016/j.cognition.2009.10.005)

9. Connell L, Lynott D. 2014 I See/Hear What You Mean: Semantic Activation in Visual Word Recognition Depends on Perceptual Attention. J. Exp. Psychol. Gen. 143, 527-533. (doi:10.1037/a0034626)

10. Wellsby M, Siakaluk PD, Owen WJ, Pexman PM. 2011 Embodied semantic processing: The body-object interaction effect in a non-manual task. 3, 1-14. (doi:10.1515/langcog.2011.001)

11. Zwaan RA, Taylor L. 2006 Seeing, acting, understanding: Motor resonance in language comprehension. J. Exp. Psychol. Gen. 135, 1-11. (doi:10.1037/0096-3445.135.1.1)

12. Simmons WK, Ramjee V, Beauchamp MS, McRae K, Martin A, Barsalou LW. 2007 A common neural substrate for perceiving and knowing about color. Neuropsychologia 45, 2802-2810. (doi:10.1016/j.neuropsychologia.2007.05.002)

13. Boulenger V, Hauk O, Pulvermüller F. 2009 Grasping Ideas with the Motor System: Semantic Somatotopy in Idiom Comprehension. Cereb. Cortex N. Y. NY19, 1905-1914. (doi:10.1093/cercor/bhn217)

14. Buxbaum L, Saffran EM. 2002 Knowledge of object manipulation and object function: dissociations in apraxic and nonapraxic subjects. Brain Lang. 82, 179-199. (doi:10.1016/S0093-934X(02)00014-7)

15. Hyder R, Højlund A, Jensen M, Johnsen EL, Østergaard K, Shtyrov Y. 2021 STN-DBS affects language processing differentially in Parkinson's disease: Multiple-case MEG study. Acta Neurol. Scand. 144, 132-141. (doi:10.1111/ane.13423)

16. Connell L, Lynott D. 2016 Do we know what we're simulating? Information loss on transferring unconscious perceptual simulation to conscious imagery. J. Exp. Psychol. Learn. Mem. Cogn. 42, 1218-1232. (doi:10.1037/xIm0000245)

17. Shtyrov Y, Butorina A, Nikolaeva A, Stroganova T. 2014 Automatic ultrarapid activation and inhibition of cortical motor systems in spoken word comprehension. Proc. Natl. Acad. Sci. 111, E1918-E1923. (doi:10.1073/pnas.1323158111)

18. Vukovic N, Feurra M, Shpektor A, Myachykov A, Shtyrov Y. 2017 Primary motor cortex functionally contributes to language comprehension: An online rTMS study. Neuropsychologia96, 222-229. (doi:10.1016/j.neuropsychologia.2017.01.025)

19. Vukovic N, Shtyrov Y. 2019 Learning with the wave of the hand: Kinematic and TMS evidence of primary motor cortex role in category-specific encoding of word meaning. Neuro/mage 202, 116179 (doi:10.1016/j.neuroimage.2019.116179)

20. Barsalou LW, Wiemar-Hastings K. In press. Situating Abstract Concepts. In Grounding Cognition: The Role of Perception and Action in Memory, Language, and Thinking (eds D Pecher, RA Zwaan), pp. 129-163. Cambridge: Cambridge University Press.

21. Paivio A. 1990 Mental Representations: A Dual Coding Approach. New York: Oxford University Press.

22. Vigliocco G, Meteyard L, Andrews M, Kousta S. 2009 Toward a theory of semantic representation. Lang. Cogn. 1, 219-247. (doi:10.1515/LANGCOG.2009.011)

23. Connell L, Lynott D. 2012 Strength of perceptual experience predicts word processing performance better than concreteness or imageability. Cognition 125, 452-465. (doi:10.1016/j.cognition.2012.07.010)

24. Speed LJ, Brybaert M. 2021 Dutch sensory modality norms. Behav. Res. Methods (doi:10.3758/s13428-021-01656-9)

25. Zdrazilova L, Pexman PM. 2013 Grasping the invisible: Semantic processing of abstract words. Psychon. Bull. Rev. 20, 1312-1318. (doi:10.3758/s13423-013-0452-x)

26. Banks B, Wingfield C, Connell L. 2021 Linguistic Distributional Knowledge and Sensorimotor Grounding both Contribute to Semantic Category Production. Cogn. Sci. 45, e13055. (doi:10.1111/cogs.13055)

27. Niedenthal PM, Winkielman P, Mondillon L, Vermeulen N. 2009 Embodiment of emotion concepts. J. Pers. Soc. Psychol. 96, 1120-1136. (doi:10.1037/a0015574)

28. Moseley R, Carota F, Hauk 0, Mohr B, Pulvermüller F. 2012 A Role for the Motor System in Binding Abstract Emotional Meaning. Cereb. Cortex 22, 1634-1647. (doi:10.1093/cercor/bhr238)

29. Borghi A, Flumini A, Cimatti F, Marocco D, Scorolli C. 2011 Manipulating Objects and Telling Words: A Study on Concrete and Abstract Words Acquisition. Front. Psychol. 2, 15. (doi:10.3389/fpsyg.2011.00015)

30. Borghi AM, Zarcone E. 2016 Grounding Abstractness: Abstract Concepts and the Activation of the Mouth. Front. Psychol. 7, 1498. (doi:10.3389/fpsyg.2016.01498)

31. Villani C, Lugli L, Liuzza MT, Borghi AM. 2019 Varieties of abstract concepts and their multiple dimensions. Lang. Cogn. 11, 403-430. (doi:10.1017/langcog.2019.23)

32. Badets A, Pesenti M. 2010 Creating number semantics through finger movement perception. Cognition 115, 46-53. (doi:10.1016/j.cognition.2009.11.007)

33. Desai RH, Reilly M, van Dam W. 2018 The multifaceted abstract brain. Philos. Trans. R. Soc. B Biol. Sci. 373, 20170122. (doi:10.1098/rstb.2017.0122)

34. Fischer MH, Shaki S. 2018 Number concepts: abstract and embodied. Philos. Trans. R. Soc. B Biol. Sci. 373, 20170125. (doi:10.1098/rstb.2017.0125)

35. Ranzini M, Lugli L, Anelli F, Carbone R, Nicoletti R, Borghi A. 2011 Graspable Objects Shape Number Processing. Front. Hum. Neurosci. 5, 147. (doi:10.3389/fnhum.2011.00147)

36. Borghi AM, Barca L, Binkofski F, Tummolini L. 2018 Varieties of abstract concepts: development, use and representation in the brain. Philos. Trans. R. Soc. B Biol. Sci. 373 , 20170121. (doi:10.1098/rstb.2017.0121)

37. Kloos H, Sloutsky VM. 2008 What's behind different kinds of kinds: Effects of statistical density on learning and representation of categories. J. Exp. Psychol. Gen. 137, 52-72. (doi:10.1037/00963445.137.1.52)

38. Battig WF, Montague WE. 1969 Category norms of verbal items in 56 categories A replication and extension of the Connecticut category norms. J. Exp. Psychol. 80, 1-46. (doi:10.1037/h0027577)

39. Banks B, Connell L. 2021 Category Production Norms for 117 Concrete and Abstract Categories. (doi:10.31234/0sf.io/7peqx)

40. Lynott D, Connell L, Brysbaert M, Brand J, Carney J. 2020 The Lancaster Sensorimotor Norms: multidimensional measures of perceptual and action strength for 40,000 English words. Behav. Res. Methods52, 1271-1291. (doi:10.3758/s13428-019-01316-z)

41. Banks B, Connell L. 2021 Data from: Multidimensional Sensorimotor Grounding of Concrete and Abstract Categories. Open Sci. Framew. (doi:10.17605/0SF.I0/N6SGR)

42. Capitani E, Laiacona M, Mahon B, Caramazza A. 2003 What are the facts of semantic category-specific deficits? A critical review of the clinical evidence. Cogn. Neuropsychol.20, $213-261$. (doi:10.1080/02643290244000266)

43. Barsalou LW, Dutriaux L, Scheepers C. 2018 Moving beyond the distinction between concrete and abstract concepts. Philos. Trans. R. Soc. B Biol. Sci.373, 20170144. (doi:10.1098/rstb.2017.0144)

44. Warrington EK, McCarthy RA. 1987 Categories of knowledge. Further fractionations and an attempted integration. Brain J. Neurol. 110 ( Pt 5), 1273-96.

45. Hodges JR, Patterson K, Oxbury S, Funnell E. 1992 Semantic dementia. Progressive fluent aphasia with temporal lobe atrophy. Brain J. Neurol. 115 ( Pt 6), 1783-1806. (doi:10.1093/brain/115.6.1783)

46. Breedin SD, Saffran EM, Coslett HB. 1994 Reversal of the concreteness effect in a patient with semantic dementia. Cogn. Neuropsychol. 11, 617-660. (doi:10.1080/02643299408251987)

47. Wingfield C, Connell L. 2021 Sensorimotor distance: A fully grounded measure of semantic similarity for 800 million concept pairs. (doi:10.31234/osf.io/fq53w)

48. Gentner D, Kurtz KJ. 2005 Relational categories. In Categorization inside and outside the laboratory: Essays in honor of Douglas L. Medin, pp. 151-175. Washington, DC, US: American Psychological Association. (doi:10.1037/11156-009)

49. Lupyan G, Mirman D. 2013 Linking language and categorization: Evidence from aphasia. Cortex 49, 1187-1194. (doi:10.1016/j.cortex.2012.06.006) 